\title{
Multilinguales
}

\section{Écriture, cadre, contexte}

Writing, Frame, Context

\section{Isabelle Delcambre}

\section{(2) OpenEdition}

Journals

Édition électronique

URL : http://journals.openedition.org/multilinguales/2168

DOI : $10.4000 /$ multilinguales. 2168

ISSN : 2335-1853

\section{Éditeur}

Université Abderrahmane Mira - Bejaia

\section{Édition imprimée}

Date de publication : 1 décembre 2013

Pagination : $9-25$

ISSN : 2335-1535

\section{Référence électronique}

Isabelle Delcambre, «Écriture, cadre, contexte », Multilinguales [En ligne], 2 | 2013, mis en ligne le 01 décembre 2013, consulté le 17 septembre 2019. URL : http://journals.openedition.org/multilinguales/ 2168 ; DOI : 10.4000/multilinguales. 2168

Ce document a été généré automatiquement le 17 septembre 2019.

\section{(c) (i) (3)}

Multilinguales est mise à disposition selon les termes de la Licence Creative Commons Attribution Pas d'Utilisation Commerciale - Pas de Modification 4.0 International 


\title{
Écriture, cadre, contexte
}

\author{
Writing, Frame, Context
}

\author{
Isabelle Delcambre
}

1 La notion de contexte a été interrogée dans de très nombreux domaines de recherche, elle reste néanmoins souvent prise au piège du sens commun. Le contexte, c'est « ce dans quoi » on se trouve lorsqu'on produit un discours ou un énoncé. Ce sens premier, spatial ou évènementiel, fait souvent obstacle à une analyse fine des multiples sens et usages de cette notion. La recherche didactique sur l'écriture a montré combien le/les contextes (physiques, sociaux, institutionnels, etc.) pèse(nt) sur la pratique scripturale dans l'univers scolaire (Reuter, 1996: 59). Très tôt, les analyses linguistiques ont éprouvé le besoin de différencier le contexte d'un énoncé (au sens spatial ou évènementiel, au minimum) du co-texte de son apparition en discours, de son environnement immédiat.

2 La notion de contexte est parfois proche de celle de cadre. Dans le modèle SPEAKING de D.H. Hymes que l'on peut considérer comme l'une des premières descriptions du contexte dans une perspective d'ethnographie de la communication, le mot setting qui est généralement traduit par "cadre», est un élément constitutif du contexte, au même titre que les participants et les buts (Kerbrat-Orecchioni, 1990 : 77).

3 Les sciences cognitives ont amplement utilisé la notion de frame pour désigner les cadres stéréotypiques qui «structurent l'univers mental d'un individu en relation avec une action dans un contexte déterminé » (de Nuchèze \& Colletta, 2002:24). De même, dans une perspective de sociologie interactionniste, Goffman intitule un de ses ouvrages Frame Analysis: An Essay on the Organization of Experience; titre traduit par Les cadres de l'expérience. Et bien d'autres. Ces cadres, de nature cognitive ou sociale, participent à la construction du contexte dans l'activité de l'individu.

4 Je ferai, dans cet article, une rapide mise au point sur certains débats autour de la notion de contexte, dans différents domaines de recherche, dont les didactiques qui utilisent cette notion en contraste avec celle de milieu. Puis je montrerai, en m'appuyant sur les travaux d'E. Goffman, comment la notion de cadre peut être considérée comme l'équivalent d'une mise en contexte, notamment disciplinaire, des 
recherches scientifiques. Et je terminerai par une réflexion sur ce que j'appelle les cadres discursifs, à savoir les cadres construits dans et par le discours, en tentant de montrer l'intérêt de cette notion pour l'analyse de l'écriture, particulièrement de l'écriture de recherche. Qu'est-ce que construire un cadre théorique (ou méthodologique) dans un mémoire ou dans une thèse? Quelles opérations discursives attestent de ce qu'un cadre a été construit? Quelles variations sont observables dans les écrits des chercheurs eux-mêmes?

\section{Contexte : une notion en débat}

\section{Le contexte : un contenant?}

5 Le sens commun et, au-delà, l'usage premier de cette notion en psychologie sociale du développement réfère le contexte à l'entour d'une activité, d'une interaction, d'une production verbale. Ce dans quoi, pour reprendre ma formule triviale de l'introduction, est situé le sujet que le chercheur observe ou dont il analyse les productions. Comme le montre Michèle Grossen (2001) ${ }^{1}$, dans les années 1970, une équipe de psychologues travaillant avec Margaret Donaldson met au jour le fait que dans les situations de tests (dans des dispositifs similaires aux dispositifs piagétiens), les réponses sont influencées par le sens que l'enfant tente de donner à la situation, à la consigne ou aux interprétations qu'il fait des attentes du chercheur, etc. En gros, ses réponses sont influencées par le contexte dans lequel les questions sont posées.

6 À partir de là, de très nombreuses recherches en psychologie du développement ont exploré le rôle du contexte dans ce type de situations : que ce soit la formulation des consignes, les connaissances préalables de l'enfant (sa familiarité avec le matériel utilisé), les attentes qu'a l'enfant concernant la tâche à effectuer, l'image de soi (l'enfant se sent-il capable ou pas de réaliser la tâche, est-il intéressé, se sent-il concerné par elle? etc.), les rôles endossés par l'adulte (se présente-t-il comme quelqu'un qui cherche à aider l'enfant ou à l'évaluer?) ou encore, pour finir, par le lieu de passation du test.

7 Ces recherches, tout en mettant en évidence l'importance du contexte dans les réponses données par le sujet, reposent sur le postulat méthodologique que ces effets de contexte sont comme des variables et qu'elles pourraient/devraient être neutralisées par une recherche bien pensée qui voudrait accéder aux capacités cognitives intrinsèques, celles qui seraient indépendantes de tout contexte (Grossen, 2001 : 64). Le contexte apparaît bien ici comme un contenant, comme ce qui englobe l'expérimentateur et le sujet observé, les cognitions du sujet restant malgré tout à atteindre indépendamment de ces variables.

8 De la même manière, dans les analyses linguistiques et pragmatiques, le contexte, qui est souvent l'objet de grandes interrogations, peut être décrit comme un feuilletage de niveaux emboîtés les uns dans les autres. C'est le cas de la typologie des contextes élaborée par Françoise Armangaud (1985). Située dans le cadre d'une interaction, cette typologie déroule de l'extérieur vers l'intérieur, quatre contextes différents: le contexte référentiel ou situationnel (le plus extérieur, l'environnement physique), le contexte paradigmatique (à savoir le discours lui-même), le contexte interactionnel (c'est-à-dire les contraintes de l'interlocution, les enchaînements de tours de parole et d'actes de langage, etc.) et le contexte présuppositionnel (qui renvoie aux dimensions 
épistémiques de l'échange et à tout ce que chacun croit, pense, croit que l'autre pense, etc.).

Il s'agit bien de niveaux différents de contexte, dont le discours semble entouré.

10 On retrouve cet emboitement de niveaux dans la partition micro/macro/meso, fréquemment utilisé dans les recherches en sociologie et en économie et parfois reprise en éducation. Le macro-système renvoie au contexte global, social, politique, culturel d'une action éducative. C'est le niveau où s'élaborent les finalités éducatives. Le mésosystème réfère au type d'institution ou de dispositif de formation qui élabore des objectifs généraux pour une intervention éducative et le micro-système se rapproche de la situation éducative concrète; il concerne les ressources matérielles et humaines, les conditions spatio-temporelles de l'action d'enseignement/apprentissages. Le niveau de décision est ici celui des enseignants et des formateurs.

\section{Le contexte : un produit?}

Pour revenir à l'étude de $\mathrm{M}$. Grossen, elle montre ensuite que la conception du contexte comme contenant en psychologie, ne prend pas en compte ce qui pourrait résulter des interactions entre adulte et enfant, de la construction de la situation par les interactants eux-mêmes.

12 Les courants de l'interactionnisme social qui s'appuient sur les travaux de Vygotsky s'attachent à décrire et à comprendre la dynamique des interactions entre les protagonistes d'une situation. Ces travaux montrent que «le contexte ne peut pas être simplement défini comme un ensemble de caractéristiques externes, car la pertinence qu'elles revêtent pour les interactants varie au cours même de l'interaction » (Grossen, $2001: 65$ ).

Certains événements de l'interaction sont chargés de pertinence à un moment donné, notamment en fonction des connaissances supposées partagées entre les interactants, et repoussent à l'arrière-plan les autres événements de la situation. Ces événements, sur lesquels les interactants focalisent leur attention ensemble et en même temps et sur lesquels ils doivent s'accorder, produisent le contexte de leur action, à ce moment-là : "Les éléments concrets de la situation dans laquelle se trouvent les sujets apparaissent alors comme des ressources susceptibles ou non d'être prises en compte par les interactants pour donner un sens aux évènements et à leurs interactions " (Grossen, 2001: 66). Le contexte est alors considéré comme le produit des interactions entre participants ; résultat instable et toujours changeant; le contexte n'est plus un contenant, il devient alors, en quelque sorte, un des contenus même de l'interaction. Cette perspective est partagée par les linguistes, notamment les spécialistes de l'analyse des interactions verbales, comme Catherine Kerbrat-Orecchioni (1990): «Donné à l'ouverture de l'interaction, le contexte est en même temps construit dans et par la façon dont celle-ci se déroule; définie d'entrée, la situation est sans cesse redéfinie par l'ensemble des événements conversationnels" (Kerbrat-Orecchioni, $1990: 106$ ).

\section{Le contexte : un système?}

La conception du contexte comme fruit d'une construction intersubjective est à son tour interrogée, en psychologie, par les courants de la cognition située. Ces travaux interrogent la dimension purement intersubjective de l'interactionnisme social et (re)donnent une place centrale aux objets (matériels ou sémiotiques) avec lesquels les 
sujets entrent en interaction: "Les objets, par leur matérialité, orientent les actions des individus et leurs interactions» (Grossen, 2001: 69). Le contexte est alors «vu comme un système cognitif qui résulte des interactions entre individus et outils et dont les éléments (humains ou non-humains) constituent potentiellement autant de ressources socio-cognitives pour les individus » (Ibid. : 70).

La conception didactique du contexte n'est pas éloignée de ce dernier courant: lorsqu'ils veulent comprendre et interpréter les conduites des élèves en situation d'apprentissage, les didacticiens se servent des notions de contexte et de milieu pour référer aux éléments matériels ou non avec lesquels les élèves entrent en relation dans une tâche donnée, "ce avec ou contre quoi l'élève agit dans une situation donnée" (Lahanier-Reuter, 2013: 49).

Le concept de "milieu», surtout théorisé en didactique des mathématiques, peut contenir des objets matériels (une équerre en mathématiques, un ballon en sport) ou sémiotiques (une frise historique, une liste des absents en maternelle, une question de l'enseignant, etc.). La notion de contexte désigne plus spécifiquement les interprétations de ces objets par les élèves : «Le contexte englobe tout ce qui fait sens dans l'univers des tâches" (Bernié, 2005: 147). Les significations attribuées à ces objets, matériels ou sémiotiques, par les élèves constituent le contexte dans lequel ils agissent. D. Lahanier-Reuter en donne un exemple particulièrement intéressant que je reprends ici (il est emprunté à Halté, 2005). Il s'agit d'un extrait de dialogue didactique :

Le maître inscrivant au tableau, soulignant et disant :

Maître : Le bûcheron coupe l'arbre. Jacques, qu'est-ce que c'est l'arbre?

Jacques : COD

Maître : Le bûcheron coupe l'arbre. Paul, qu'est-ce c'est l'arbre?

Paul : Attribut?

Jean-François Halté analyse ce dialogue en disant que "[l'élève] interprète la réitération à l'identique de la question posée par le maitre comme une évaluation négative de la réponse précédente» (2005: 65). C'est le sens que l'élève Paul donne à cette répétition de la question qui permet de comprendre son erreur. Ce sens est un élément du contexte construit par cet élève. Cette conception du contexte s'inscrit dans une théorie de l'apprentissage de type socioconstructiviste qui considère l'apprentissage comme le résultat d'un processus de "négociation de sens » (ibid.) et qui donne au langage un rôle central dans les apprentissages, ainsi qu'aux significations et valeurs accordées par les élèves aux situations proposées et aux objets rencontrés.

\section{Cadre/cadrage}

\section{Cadres et cadrages chez Goffman}

M. Grossen, dans l'article cité, évoque les notions connexes à la notion de contexte, et parmi elles, les notions de cadre et de définition de la situation, telles que théorisées en sociologie interactionniste, par Ervin Goffman et les sociologues de l'école de Chicago. La situation a une dimension objective mais elle est interprétée par l'individu qui lui donne sens et valeur.

19 La notion de définition de la situation rend compte de ces deux dimensions de la situation, objective et subjective. Elles se retrouvent dans la notion de frame, ou cadre, chez E. Goffman (notion qu'il emprunte à Gregory Bateson). Toute expérience, toute 
activité sociale, se prête, selon lui, à plusieurs versions, ou cadrages. Ces cadres, qu'il appelle primaires, fixent la représentation de la réalité, orientent les perceptions et influencent l'engagement et les conduites. En général, ils passent inaperçus et sont partagés par toutes les personnes en présence :

Dans nos sociétés occidentales, identifier un événement parmi d'autres, c'est faire appel, en règle générale, et quelle que soit l'activité du moment, à un ou plusieurs cadres ou schèmes interprétatifs que l'on dira primaires parce que, mis en pratique, ils ne sont pas rapportés à une interprétation préalable ou 'originaire'. Est primaire un cadre qui nous permet, dans une situation donnée, d'accorder du sens à tel ou tel de ses aspects, lequel autrement serait dépourvu de signification. (Goffman, 1967/1974 : 30 sq)

Goffman prend l'exemple d'un phénomène comme le lever du soleil: il peut être interprété comme un phénomène naturel, dans un cadre naturel; si ce phénomène amène à baisser le store, on peut dire qu'il est aussi interprété dans un cadre social, l'action de baisser le store étant "pilotée» par une intention humaine, celle de se protéger.

21 À l'école, des objets comme les cahiers, les copies, les couleurs de soulignement, les modes d'inscription de la date, les formes de marge, etc., participent de la construction du cadre scolaire, incarnent des attentes, des usages, des formes d'organisation de la connaissance, etc. et doivent être identifiés en tant que tel par les élèves (Rothier-Bautzer, 1998). Ces objets scolaires ont été depuis travaillés en didactique comme un des lieux, un des supports où se construit la conscience disciplinaire des élèves (Cohen-Azria, Lahanier-Reuter \& Reuter, sous presse) dont on sait qu'elle est en partie liée à la réussite à l'école. Les disciplines peuvent être considérées comme des cadres d'interprétation des activités auxquelles les élèves sont confrontés.

Il faut distinguer les cadres qui sont des schémas interprétatifs de l'activité de « cadrage qui consiste à mettre en relation ce qu'on va dire avec le champ de significations possibles, en identifiant la zone de pertinence à l'intérieur de laquelle on a l'intention de communiquer quelque chose à quelqu'un » (Goffman, 1981/1987).

Pour simplifier, on peut dire que le cadrage est une activité, un travail, alors que le cadre est ce en fonction de quoi on fait un cadrage ou ce qui va résulter de l'activité de cadrage. Le contexte n'étant ni stable ni déterminé complètement à l'avance, comme je l'ai précisé ci-dessus, est soumis à des opérations de cadrage (ou de recadrage) constantes.

\section{Cadres disciplinaires ou épistémologiques}

La notion de cadre intéresse également l'épistémologie. L'histoire des idées, des concepts ou des savoirs scientifiques permet de comprendre comment nous sommes parvenus au niveau de connaissance actuel.

Ainsi Gaston Bachelard (1949) s'attache à la construction historique de concepts scientifiques, comme la rotation de la terre autour du soleil, il décrit comment ce phénomène a été décrit, interprété, expliqué d'Aristote et Ptolémée, jusqu'à Copernic, Galilée et Newton. Il montre que ces élaborations théoriques remettent en cause le phénomène de construction linéaire des connaissances et souligne l'importance du cadre de référence mis en œuvre au cours de la structuration du savoir. Le chercheur est pris dans le processus de découverte de nouvelles idées, son cadre de référence, 
constitué par ce que Bachelard nomme ses propres représentations ou ses connaissances préalables, joue un rôle masquant.

Le savoir ne s'acquiert pas d'emblée par observation, il s'élabore à partir de connaissances déjà en place; c'est par un processus de prise de recul, de décantation que s'engendre une autre approche de la réalité.

Ainsi, pour l'épistémologie, le savoir scientifique est une construction de l'esprit qui est fondée en confrontation avec la réalité et par rapport aux évidences antérieures. Pour reprendre les formulations goffmanniennes, on pourrait dire que la construction du savoir est le produit d'une longue série de cadrages (recadrages et décadrages, si l'on veut bien m'autoriser ce néologisme).

En didactique, on considère que les disciplines (scolaires, universitaires ou de recherche) sont des cadres de référence, de nature épistémologique (théorique et méthodologique), qui entrent en interaction avec un certain nombre de pratiques, notamment d'écriture. L'idée centrale ici est que les pratiques langagières ne sont ni transparentes ni transversales aux différents contextes. La capacité langagière est fortement (peut-être indissolublement liée) aux contextes où elle se déploie, aux cadres (sociaux, culturels, épistémologiques) qui sont mobilisés comme ressources pour ce déploiement. Sans que cette relation soit déterministe, d'ailleurs : il y a interaction entre cadres et pratiques : les pratiques d'écriture contribuent à construire les cadres disciplinaires tout autant qu'elles sont inscrites elles-mêmes dans des cadres (disciplinaires, génériques, discursifs, etc.).

29 J'en prendrai un exemple extrait de la thèse que Nathalie Constant a soutenue en didactiques et qui interroge les pratiques de description en français et en mathématiques, à trois niveaux scolaires différents (fin de l'école primaire, fin du collège, fin du lycée). Je ne retiens ici que deux exemples produits par le même élève de $3^{\circ}$, en réponse à la consigne "décris un pavé » (consigne spécialement pensée pour avoir du sens dans les deux disciplines (Constant-Berthe, 2006) :

Description d'un pavé en français

Mon pavé est orange. Il embaume le frigo de son doux parfum. Il a les bouts arrondis, et sa croûte est épaisse. Son odeur n'empêche pas son goût. Il est moelleux à l'intérieur. Il est assez petit, assez gros. Il trône au milieu des autres, comme un roi dans son palais. Même la cloche ne l'empêche pas d'atteindre nos narines. Après le maroilles, c'est lui que je préfère.

Description d'un pavé en mathématiques

Mon pavé est haut de 3 mètres, long de 8 mètres et large de 4 mètres. Ses arrêtes sont ou bien perpendiculaires ou bien parallèles. Les points de sa base supérieure visible sont $\mathrm{A}, \mathrm{B}, \mathrm{D}, \mathrm{H}$, dans le sens des aiguilles d'une montre. Les points de sa base inférieure sont $\mathrm{EFGC}$ dans le sens des aiguilles d'une montre.

Les deux écrits répondent à des attentes spécifiques, perçues par les élèves, ils permettent d'identifier des représentations différentes des textes attendus dans chacune des disciplines. Au-delà du lexique (arrêtes, perpendiculaire, parallèle, base supérieure, inférieure) et d'autres formes d'écriture spécifiques aux mathématiques (comme le fait de désigner les sommets par des lettres, en indiquant le sens de lecture/ écriture), les propriétés du pavé retenues en mathématiques diffèrent sensiblement de celles retenues en français : d'un côté, hauteur, longueur, largeur ; de l'autre, couleur, forme globale («arrondi»), odeur, goût, consistance. Ce sont pour l'essentiel des propriétés renvoyant à la perception sensorielle; de plus l'usage d'une comparaison, l'organisation générale du texte en forme de devinette (on a bien un indice, dans le 
nom de ce célèbre fromage du nord de la France, mais c'est insuffisant pour identifier clairement quel fromage l'élève évoque dans son texte), la phrase finale en forme de chute, et la présence d'un énonciateur (qui fait part d'un ressenti «c'est lui que je préfère ») réfèrent à des "habitudes d'écriture », en fait des cadres culturels, spécifiques à cette discipline.

31 L'enjeu discursif des deux textes est également complètement opposé : en français, il s'agit de faire voir l'objet, de créer une image mentale, en mathématiques, de donner des indications suffisantes pour construire une représentation géométrique du pavé ${ }^{2}$.

Ce dispositif de recherche permet d'identifier comment jouent les cadres disciplinaires, comment ils donnent forme aux pratiques langagières. On voit, par ailleurs, comment la notion de cadre est ici mobilisée « en actes ", elle est bien évidemment non explicitée par l'élève qui écrit (et probablement non conscientisée).

\section{Cadres discursifs}

J'en viens à la dernière section de cet article où je vais rendre compte de quelques considérations sur l'écriture de recherche en lien avec la notion de cadre. De mon point de vue, ce qu'on appelle le cadre théorique dans un écrit de recherche équivaut peu ou prou à l'élaboration d'un contexte de réception (connaissances partagées, mémoire commune), à l'élaboration d'un cadre primaire à partager avec le lecteur.

C'est le contexte dans lequel un énoncé (à savoir le mémoire, la thèse) peut prendre sens, c'est l'ensemble des théories ou des questionnements qui lui donnent sa pertinence, sa valeur. Le cadre théorique s'apparente ainsi à ce qu'on appelle par ailleurs la problématique d'une recherche: dans quel cadre sont situées les questions qu'on pose?

Construire un cadre théorique va de pair, dans le travail de l'apprenti chercheur, avec le repérage des cadres théoriques des textes qu'il lit. Ainsi, un étudiant de licence, résumant un article d'A.M. Chartier sur les usages de l'oral dans différentes cultures scolaires, écrit "certains considèrent la prise de parole comme un signe de spontanéité, d'autres comme un signe de mauvaise éducation ", là où l'auteur avait écrit "alors que nous voyons dans la prise de parole spontanée un signe de vivacité intellectuelle et de confiance relationnelle, bref, de bonne santé, on y voit, dans d'autres temps et d'autres lieux, le signe d'une mauvaise éducation ou d'une conduite très perturbée ». L'étudiant produit par sa reformulation un changement de perspective que l'on peut identifier à un recadrage du texte source : il efface le cadre historico-culturel construit par l'auteur et le remplace par un cadre s'apparentant à la psychologie ordinaire, marquée de relativisme.

Le cadre théorique pose problème à l'étudiant en écriture comme en lecture, comme je l'avais analysé, dans un travail précédent (Delcambre, 2004). Je m'attacherai cependant plutôt ici aux questions liées à la production d'un cadre théorique. Que veut dire écrire son/un cadre théorique ? Et au-delà des tâches d'élaboration d'un cadre, liées à la connaissance du champ, quelles opérations discursives mettre en œuvre pour rendre visible/lisible le fait de construire un cadre théorique? 


\section{Observation d'écrits de recherche}

37 L'observation d'écrits de chercheurs permet de se faire une idée des procédures possibles. En effet, quand dans un ouvrage, un article, un chercheur affiche qu'il va procéder dans ce passage à un cadrage, il peut être intéressant de voir comment il fait, à quelles opérations discursives ce cadrage correspond. Je propose ainsi d'observer un extrait du livre de B. Charlot, E. Bautier et J.Y. Rochex, École et savoir dans les banlieues ... et ailleurs (1992, A. Colin) que j'ai choisi pour la diversité des opérations de cadrage qu'il présente.

Le sommaire fait apparaître une sous-partie intitulée "Cadrage théorique et méthodologique » (p. 184-188) qui apparaît tardivement dans l'ouvrage (cela s'explique par le fait qu'il précède la présentation des résultats d'une recherche spécifique sur les apprentissages scolaires à l'école élémentaire, et qu'il est donc précisément articulé à cette recherche). Que font les chercheurs dans ce « cadrage »?

Je liste ici, dans l'ordre du texte, les opérations discursives mises en œuvre, très succinctement identifiées aux marqueurs discursifs identifiables en début de paragraphe :

- une définition: «nous considérons les apprentissages scolaires comme le produit de différents processus »;

- une dissociation conceptuelle : «Parler de disciplines scolaires ne signifie pas pour autant que l'on pose la question des apprentissages en termes de connaissances disciplinaires »;

- une justification des choix méthodologiques effectués : «Compte-tenu de cette conception des processus qui sous-tendent les apprentissages, en particulier les apprentissages scolaires, nous avons recueilli des données larges et hétérogènes »;

- un rappel des sources théoriques: "Nous reprenons, dans ce domaine, les conceptions de Vigotsky, de Bruner et de Bernstein quant aux relations entre langage, socialisation et cognition »;

- une annonce des résultats: "Nous présentons ci-après successivement les analyses concernant les rapports des élèves de CP à l'école, puis celles ... » ;

- une dissociation entre champs de recherches: «Nous ne nous situons cependant pas dans une perspective didactique, nous tentons de comprendre ce qui, avec certains élèves, peut produire des effets négatifs tant sur les apprentissages que sur la construction de leur rapport au savoir ";

- une réponse anticipée à une interrogation méthodologique possible du lecteur: "On pourrait s'interroger sur la validité d'une étude qui repose sur le langage d'élèves dont on souligne habituellement la faiblesse langagière (...). On pourrait discuter du caractère peu représentatif d'un travail qui porte sur une centaine d'élèves ».

On pourrait dire que ces chercheurs balisent la zone de pertinence de leur recherche (pour reprendre Goffman); ils construisent une "définition de la situation » dans laquelle le lecteur doit se situer pour comprendre et interpréter ce qu'ils disent. Par exemple, ne pas les prendre pour des didacticiens, intéressés aux contenus disciplinaires. Si l'on veut donner du sens au terme de cadre théorique ici, on pourrait dire qu'il réalise une métaphore spatiale (ce qu'exprime aussi le terme de «champ " présenté par Michèle Grossen (2001: 61) comme une notion voisine de celle de contexte).

41 Certes, cette analyse est extrêmement limitée et ne dit rien de toutes les autres opérations discursives ou énonciatives possibles. J'ai retenu cet exemple pour sa 
diversité comme je le disais plus haut, mais aussi pour illustrer une démarche de formation qui repose sur des principes depuis longtemps établis en didactique de l'écriture : lire pour (mieux) écrire.

\section{Les recherches rhétoriques sur l'écrit universitaire (de recherche)} systématiser ce genre d'analyse des écrits, à partir principalement d'observations linguistiques et énonciatives.

Ce champ de recherche, particulièrement développé dans l'équipe Lidilem à Grenoble 3 (voir par exemple Lidil, 2010), est ancré dans une perspective de linguistique du discours et une méthodologie de linguistique de corpus (voir le site Scientext, porté par la Maison des Sciences de l'Homme-Alpes). Il s'agit de décrire linguistiquement les fondements des communautés discursives universitaires avec deux approches: une approche rhétorique du discours scientifique et des analyses énonciatives. L'étude de la construction discursive du savoir scientifique et de ses dimensions argumentatives repose sur l'idée que les discours scientifiques ne sont ni neutres ni objectifs, qu'ils comportent des formes de subjectivité et de persuasion, ce qui entre en cohérence avec une conception constructiviste de la science (Latour et Fabbri, 1977).

Francis Grossmann, dans la présentation au numéro 2010/3 de la revue électronique Anthropologie des connaissances, met en évidence deux grandes caractéristiques de l'écrit de recherche: sa forte dimension intertextuelle et une double contrainte d'effacement énonciatif et de construction d'un point de vue d'auteur.

Ces caractéristiques placent les étudiants dans des tensions qui ne facilitent pas l'écriture, comme je l'ai montré récemment (Delcambre, 2012).

La perspective énonciative sur l'écriture du cadre théorique, ou du moins une des manifestations du cadre que représente l'introduction, a été travaillée, il y a longtemps, par Violaine de Nuchèze (1998). Je la présente rapidement, car elle permet d'aborder une autre dimension du cadre que celles que j'ai présentées ci-dessus, que j'ai appelé le cadre figuratif (Delcambre, $2004: 48$ ).

À partir d'un corpus d'articles en Sciences du langage, de Nuchèze (1998) décrit les « routines scripturales » de l'introduction et dégage cinq types de cadrage :

- le premier se présente sous la forme d'énoncés généralisants, de discours d'autorité, masquant l'énonciateur du texte ou le protégeant d'un contre-discours potentiel: «Les linguistes s'accordent en général ... », "Traditionnellement, on attribue à...», " Nous partirons de la définition la plus courante...»;

- le second est auto-centré et polémique : «Pour éviter une démarche aussi paradoxale, nous proposons dans cet article de remettre en cause.. »;

- le troisième est également auto-centré, mais ni polémique ni généralisant : « L'objectif de cet article... », « Il s'agira ici d'explorer... », « Dans cet article, nous essaierons de démontrer... »;

- le quatrième consiste en une planification de l'article à venir : «Dans une première partie... En conclusion... »;

- le cinquième se présente comme des conduites d'auto-défense : "Je ne définirai pas ici... ", «Je ne m'intéresserai pas ici... », « Faute de place, je ne reviendrai pas sur... »".

Le tout dernier cadrage s'apparente pour moi à un acte de figuration (Goffmann 1967/1974). Selon Goffmann, la règle fondamentale que doit respecter tout individu en 
interaction avec d'autres est de préserver sa face et celle de ses partenaires. C'est la condition de possibilité de toute interaction. Pour assurer le respect de sa face et de celle des autres, un travail de figuration est nécessaire, pour éviter de compromettre les faces: c'est le tact, le savoir-vivre ou encore la diplomatie. Dans l'écrit, l'anticipation des critiques potentielles permet au scripteur de préserver sa face, de lui conserver une «valeur sociale positive" (Goffmann, 1967/1974: 9). D'où le terme de cadrage figuratif que j'ai proposé.

Je voudrai terminer cette longue digression sur le cadre discursif en m'appuyant sur un autre travail de la même équipe grenobloise qui a mis en œuvre une démarche empirique très proche. Françoise Boch, Francis Grossmann et Fanny Rinck (2009) ont en effet analysé des articles de recherche en linguistique pour identifier, dans les introductions, la circulation des discours qui ancrent l'objet de recherche dans un contexte théorique, pour reconstituer les discours circulant autour de l'objet de recherche. Pour penser toujours avec Goffmann, le cadre théorique construit un contexte de réception, un cadre primaire, dirait Goffmann. Mais Boch, Grossmann et Rinck ont observé différentes façons de faire.

La circulation des discours peut se présenter comme une diffusion des théories et des concepts, une "migration" des concepts, sur des emprunts théoriques, sur une continuité ou des transferts d'un champ à l'autre. Le cadrage dans ce cas s'effectue en référence aux savoirs déjà là, sans opposition aux discours qui circulent. En voici un exemple (je souligne les éléments de discours sur lesquels s'appuie l'analyse) ${ }^{5}$ :

En France, depuis plus d'une quinzaine d'années, de nombreuses études ont entrepris de décrire, selon diverses modalités, des variétés de français parlées par des adolescents urbains. Au-delà de son inscription dans ce courant descriptif, le propos de cet article est également d'examiner le degré de spécificité des pratiques décrites.

51 La circulation des discours peut aussi se présenter comme un renouvellement qui suppose des déplacements, une altération dans la reprise des discours, une réinterprétation; l'interdiscours repose sur un travail de reformulations (une terminologie que l'on récuse comme inadéquate) associé à une rhétorique du neuf (proposition d'une nouvelle définition et/ou d'un nouveau terme). Le cadrage met en œuvre alors une démarcation entre l'apport personnel du chercheur et la doxa ou les travaux antérieurs :

Dans le cadre des études sur la politesse linguistique, la politesse des actes de discours, et surtout des actes de discours directifs (requêtes, ordres, etc.), est souvent associée implicitement à leur caractère plus ou moins indirect. La relation entre la politesse d'un énoncé et l'indirection a déjà fait couler des flots d'encre. Le but du présent article est de faire le point de la situation et de remettre en question quelques conceptions discutables qui ont toujours cours. En premier lieu, il s'agira de montrer qu'il est plus judicieux de remplacer la notion d'indirection, qui renvoie à la forme linguistique de l'énoncé, par celle d'optionalité de l'énoncé (...).

52 Enfin, en référence à la notion de "niche », particulièrement mise en évidence par Swales (1990), l'interdiscours peut enfin reposer sur une synthèse des travaux existants et une légitimation de l'objet d'étude par rapport à l'état d'avancement du champ. Le chercheur montre en quoi l'apport de son étude permet de faire avancer le champ par un questionnement nouveau, il fait sa propre promotion, il crée sa « niche ».

Diverses conceptions fondent les différentes théories de la lecture. Leurs formulations jalonnent la recherche sur la communication écrite des cinquante 
dernières années. Tantôt elles concernent exclusivement la lecture de l'œuvre d'art littéraire, telles les théories de Jean-Paul Sartre, Lucien Goldmann, Hans Robert Jauss, Wolfgang Iser et l'école de Constance, Michel Picard et dans une moindre mesure d'Umberto Eco. Tantôt les théories de la lecture inclinent vers une analyse plus sociologique et institutionnelle que psychologique du lecteur d'imprimés. Ici se placent les théories déduites et illustrées de travaux empiriques de Robert Escarpit et l'école de Bordeaux, de Pierre Bourdieu, de Jean-Claude Passeron. Les premières prennent leur point de départ dans les textes, les secondes tracent leur chemin à partir du lecteur ou des groupes de lecteurs, voyageant à travers toutes sortes de textes. Aucune ne traque le braconnier évoqué par Michel de Certeau. Si toutes les théories de la lecture font du lecteur l'élément fondamental de la communication écrite, aucune ne met vraiment l'accent sur le rôle de l'affectivité dans l'implication du lecteur, ni sur la dimension psychoaffective de l'acte de lecture (...). travaux antérieurs (l'interdiscours) et la démarcation par rapport aux discours en circulation. En cela elles sont spécifiques, par rapport à celles qui ont été présentées ci-dessus. par rapport aux approches attestées. C'est pourquoi le référencement est une dimension importante de l'écriture universitaire, reconstitution de la chaîne des emprunts, tentation de l'exhaustivité et de la traçabilité, recherche de la source première d'un concept. Mais le cadrage n'est jamais neutre : il fournit une vision du champ (sélection, reformulation, etc.) qui est le choix du scripteur, une trace de son " auctorialité ». Le cadrage discursif est à la fois un comportement épistémologique et un ensemble de routines rhétoriques.

55 Pour conclure rapidement ce trop long article, je dirais, me centrant sur la notion de cadre, que l'élaboration d'un cadre théorique (dans un mémoire, dans une thèse, etc.) effectue toute une série de contextualisations de la recherche, notamment disciplinaires. Les modalités en sont essentiellement dialogiques : que ce soit par de l'interdiscours ou par des actes de figuration, le cadrage discursif actualise le deuxième sens de contexte présenté ci-dessus. Il se construit par interaction avec les discours d'autrui ou avec le lecteur potentiel. Ces deux modalités ne sont d'ailleurs pas exclusives l'une de l'autre. Au contraire, elles peuvent être cumulatives, entre elles et avec bien d'autres dimensions comme l'a montré l'analyse du texte de Charlot, Bautier et Rochex. Si l'on voulait penser les opérations de cadrage en référence avec le troisième sens de contexte, le contexte comme système d'interaction complexe avec des objets ou des outils, il faudrait probablement passer à un niveau de plus grande abstraction, en le considérant (ou du moins des morceaux rhétoriques comme l'introduction, car les opérations de cadrage se trouvent à de multiples moments différents d'un mémoire) comme un genre discursif et voir comment ce genre, à supposer qu'il en soit un, fonctionne comme un outil intellectuel dans l'élaboration d'un texte (Delcambre, 2013 : 114). 


\section{BIBLIOGRAPHIE}

ARMANGAUD F., La pragmatique, Paris, PUF, collection Que sais-je ?, 1985.

BACHELARD G., Le rationalisme appliqué. Paris, PUF, 1949.

BERNIÉ J.-P., « Les "situations différées" de Francis Ruellan : fécondité et zones d'ombres d'un espace de problématisation ", in Y. Reuter, dir., Pédagogie du projet et didactique du français. Penser et débattre avec Francis Ruellan, Villeneuve d'Ascq, Presses universitaires du Septentrion, 2005, pp. 141-168.

восн F., Grossmann F. \& Rinck F., « Le cadrage théorique dans l'article scientifique : un lieu propice à la circulation des discours ", in. J.-M. Lopez-Munoz, S. Marnette \& L. Rosier (Eds.), Actes du Colloque International Ci-dit, Québec, Nota Bene, 2009, pp. 23-42.

COHEN-AZRIA C., LAHANIER-REUTER D., REUTER, Y. (eds.), La conscience disciplinaire : les représentations des disciplines à l'école primaire, Rennes, P.U.R. (sous presse).

CONSTANT-BERTHE N., « Pourquoi et comment élaborer une consigne identique et pertinente dans deux disciplines différentes (mathématiques et français) », in M.-J. PERRIN-GLORIAN \&Y. REUTER, Les méthodes de recherche en didactiques, Villeneuve d'Ascq, Presses universitaires du Septentrion, 2006, pp. 71-84.

NUCHÈZE V., « Approche pragmatico-énonciative du discours de recherche (à l'usage des apprenants-chercheurs) ", in Lidil, $n^{\circ} 17$, Pratiques de l'écrit et modes d'accès au savoir dans l'enseignement supérieur (Dabène, M. \& Reuter, Y. dir.), 1998, pp. 25-40.

NUCHÈZE V., COLLETTA, J.-M. (éds.), Guide terminologique pour l'analyse des discours. Lexique pour l'approche pragmatique du langage, Bern, Peter Lang, 2002.

DELCAMBRE I., " Cadre et cadrage discursifs : problèmes d'écriture théorique », in Pratiques, $\mathrm{n}^{\circ}$ 121-122, Les écrits universitaires (Boch, F., Laborde-Milaa, I. \& Reuter, Y., dir), 2004, pp. 45-57. DELCAMBRE I. « Discours d'autrui et littéracies universitaires. Didactiques, $\mathrm{n}^{\circ} 2$, Actes $d u 2^{\circ}$ colloque international sur la sémiotique, la didactique et la communication de Medea ", Medea, Laboratoire de Didactique de la langue et des textes, Université Dr. Yahia Fares (Algérie), 2012, pp. 5-16.

DELCAMBRE I. « Genres », in. Reuter, Y. Dictionnaire des concepts fondamentaux des didactiques, Bruxelles, De Boeck Université, $3^{\circ}$ éd, 2013, pp. 113-118.

DELCAMBRE I., « Pratiques langagières », in Reuter, Y., Dictionnaire des concepts fondamentaux des didactiques, Bruxelles, De Boeck Université, $3^{\circ}$ éd, pp. 169-174.

GOFFMAN E., Les rites d'interaction, Paris, Éditions de Minuit (trad. frse 1974), (1967/1974).

GOFFMAN E., Façons de parler, Paris, Éditions de Minuit (trad. frse 1987), (1981/1987).

GROSSMANN, F. (éd.), L'auteur scientifique. Des rhétoriques aux épistémologies. Anthropologie des connaissances 2010/3, vol. 4, n³, 2010, [disponible sur : https://www.cairn.info/revueanthropologie-des-connaissances-2010-3.htm\%5d].

GROSSEN M., «La notion de contexte : quelle définition pour quelle psychologie ? Un essai de mise au point », in J.-P. Bernié (dir.), Apprentissage, développement et significations, Bordeaux, Presses universitaires de Bordeaux, 2001, pp. 59-76. 
HALTÉ J.-F. « Interactions : une problématique à la frontière », in J.-L. Chiss, J. David \& Y. Reuter (dir.), Didactique du français : Fondements d'une discipline, Bruxelles, De Boeck, 2005, pp. 61-76. KERBRAT-ORECCHIONI C., Les interactions verbales, T.1., Paris, Armand Colin, 1990. LAHANIER-REUTER D., « Contexte - Milieu », in Y. Reuter, Dictionnaire des concepts fondamentaux des didactiques Bruxelles : De Boeck Université, 3º́d, 2013, pp. 49-54.

LATOUR B. et Fabbri P., « La rhétorique de la science : pouvoir et devoir dans un article scientifique ", in Actes de la recherche en Sciences sociales, vol. 13, 1977, pp. 81-95.

LIDIL, $n^{\circ}$ 41, Enonciation et rhétorique dans l'écrit scientifique (F. Boch et F. Rinck, dir.), 2010.

REUTER Y., Enseigner et apprendre à écrire, Paris, ESF, 1996.

REUTER Y. « La conscience disciplinaire : Présentation d'un concept », in Education et Didactique, vol. $1, \mathrm{n}^{\circ} 2,2007$, pp. 57-71.

ROTHIER-BAUTZER E. «Le rôle des objets dans le cadrage de l'activité pédagogique : Pour une analyse contextualisée de la difficulté scolaire ", in Revue Française de Pédagogie, n 124, 1998, pp. 81-89.

SWALES J., Genre Analysis: English in Academic research Settings, Cambridge, Cambridge University Press, 1990.

\section{NOTES}

1. Je m'appuie principalement sur ce texte qui fournit de très utiles analyses pour penser les différents sens de "contexte", même si son ancrage est psychologique. Je prendrai quelques autres exemples en linguistique interactionnelle et en didactique pour élargir le propos.

2. Bien évidemment, les oppositions ne sont pas toujours aussi claires, on trouve des traces de narrativisation dans certains textes écrits pour les mathématiques, ou à l'inverse, des traces d'écriture mathématique dans des textes écrits pour le français. C'est tout l'enjeu d'une telle recherche comparative de mettre en évidence la complexité des représentations disciplinaires (ce qu'à Théodile, nous appelons la conscience disciplinaire, cf. Reuter, 2007).

3. V. de Nuchèze montre que ces formats de cadrage sont culturellement différenciés, les articles écrits par des chercheurs anglo-saxons ou allemands préférant ce cadrage spécifique et autocentré.

4. On a vu plus haut ces deux derniers procédés de cadrage à l'oeuvre dans les pages de Charlot, Bautier et Rochex.

5. Les trois exemples qui suivent sont extraits de Boch, Grossmann et Rinck, 2009.

\section{RÉSUMÉS}

La notion de contexte est l'objet de nombreuses réflexions théoriques, dans différents champs de recherche qui en établissent la variabilité de sens et d'emploi. Certains débats autour de la notion de contexte sont, ici, rapidement exposés, avant d'en venir à la notion de cadre qui n'est pas sans 
rapport avec celle de contexte. Je montrerai que les disciplines peuvent être vues comme des cadres qui sont en interaction avec les pratiques d'écriture. Et, je terminerai sur la notion de cadre discursif qui permet de penser certaines spécificités de l'écriture universitaire.

Numerous theoretical studies in different research fields establish the variability of meaning and use of the notion of context. Some debates around this notion are first briefly exposed, before coming to the notion of frame, which is not unrelated to that of context. We will show that disciplines can be seen as frames (or contexts) that are interacting with writing practices. Finally, the notion of discursive framework is useful in a didactic perspective to make students more aware of some characteristics of academic writing.

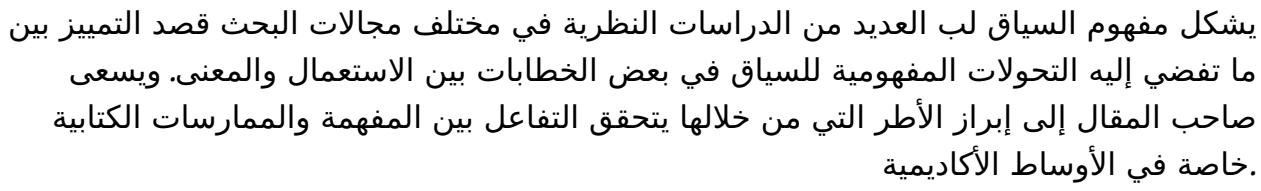

INDEX

Mots-clés : écriture universitaire, cadre/cadrage théorique, didactique

الكتابة الأكاديمية, الإطار/التأطير النظري, التعليميةفهرس الكلمات المفتاحية:

Keywords : university writing, theoretical frame/framing, didactics

\section{AUTEUR}

\section{ISABELLE DELCAMBRE}

Théodile-CIREL, EA 4354 - Université Lille3 - France 